\title{
Buffer Occupancy of Statistical Multiplexers with Periodic Interchangeable Traffic in ATM Networks
}

\author{
Zhong $\mathrm{Yu}$ and Robert J. McEliece ${ }^{1}$ \\ Dept. of Electrical Engineering, Caltech \\ Pasadena, CA 91125, U. S. A. \\ Email: zyu@systems.caltech.edu
}

\begin{abstract}
In this paper we analyze the buffer occupancy in a statistical multiplexer in ATM networks for a special type of traffic, namely, periodic interchangeable (PI) traffic. Certain generalized Ballot theorem is applied to analyze the problem. Explicit formulas for the expected buffer occupancy are derived.
\end{abstract}

\section{INTRODUCTION}

An important concept in ATM networks is the efficient sharing of link capacities through statistical multiplexing of traffic sources. Buffering is provided at a statistical multiplexer to manage traffic fluctuations when the instantaneous rate of the aggregate incoming traffic exceeds the capacity of the outgoing link. The analysis of buffer occupancy for a statistical multiplexer with burst-constrained traffic is important to maintain quality-of-service guarantees in ATM networks. In this paper, we analyze the buffer occupancy for a statistical multiplexer with a special type of burst-constrained traffic, periodic interchangeable traffic.

\section{BALlot THEOREM AND INTERCHANGEABLE RANDOM VARIABLES}

Ballot Theorem [2]. If in a ballot candidate A scores $a$ votes and candidate $B$ scores $b$ votes and if $a \geq b$, then the probability that throughout the counting the number of votes registered for $A$ is always greater than the number of votes registered for $B$ is given by

$$
P(a, b)=\frac{a-b}{a+b}
$$

provided that all possible voting records are equally likely.

Definition. Random variables $\left(X_{1}, X_{2}, \cdots, X_{M}\right)$ are cyclically interchangeable if $\left(X_{1}, X_{2}, \cdots, X_{M}\right)$ take the value $\left(x_{1}, x_{2}, \cdots, x_{M}\right)$, then they have the same probability for taking values of every cyclic permutation of $\left(x_{1}, x_{2}, \cdots, x_{M}\right)$; they are called interchangeable if they have the same probability for taking values of every permutation of $\left(x_{1}, x_{2}, \cdots, x_{M}\right)$.

Theorem (Takacs) [2]. If $\left(A_{1}, A_{2}, \cdots, A_{M}\right)$ are cyclically interchangeable random variables such as $\sum_{i=1}^{M} A_{i}=N$, then

$$
P\left\{\sum_{j=1}^{i} A_{j}<i, \forall i \in[1, M]\right\}= \begin{cases}1-\frac{N}{M} & \text { if } N<M \\ 0 & \text { otherwise }\end{cases}
$$

\footnotetext{
${ }^{1}$ This work was supported by a grant from PacBell
}

\section{Statistical Multiplexers With Periodic INTERCHANGEABLE TrAFFIC}

Consider a statistical multiplexer with an infinite buffer. Assume time is slotted and it takes a slot of time for the transmission line to transmit a cell. Suppose source $i, 1 \leq i \leq K$, generates $n_{i}$ cells within a period of $M$ slots; each source produces cells periodically with the same period $M$. The utilization factor $\rho=\frac{N}{M}<1$. Define

$$
\left\{\begin{array}{l}
Q_{k}=\text { buffer occupancy at the end of the } k \text { th slot } \\
A_{k}=\text { number of arriving cells in the } k \text { th slot, }
\end{array}\right.
$$

and $\bar{Q}$ the expected buffer occupancy. Note that $\sum_{i=1}^{M} A_{i}=$ $\sum_{i=1}^{K} n_{i}=N$. Assume random variables $A_{1}, A_{2}, \cdots, A_{M}$ are interchangeable. Then, the traffic model is called periodic interchangeable (PI) traffic, which is a $(\sigma, \rho)$ regulated traffic source with $\sigma=\rho(M-1)$. A PI traffic in which each source generates cell uniformly within a period is called uniform PI (UPI) traffic. The UPI traffic in which each source generates only one cell within a period is called the unit UPI (UUPI) traffic [1]. Applying Takacs' generalized Ballot theorem, we can prove the following theorems

Theorem 1. For a statistical multiplexer with UPI traffic,

$$
\vec{Q}=\sum_{q=1}^{N} \sum_{r=1}^{N-q} \frac{M-N+q}{M-r} \sum_{\substack{\sum_{i=1}^{K} \\
0 \leq m_{i}=q+r}} \prod_{i=1}^{K} \frac{\left(\begin{array}{c}
r \\
m_{i}
\end{array}\right)\left(\begin{array}{c}
M-r \\
m_{i}-m_{i}
\end{array}\right)}{\left(\begin{array}{c}
M \\
n_{i}
\end{array}\right)}
$$

Theorem 2. For a statistical multiplexer with UUPI traffic, when $K$ is sufficiently large,

$$
\bar{Q} \begin{cases}=\frac{1}{2}\left(\sqrt{\frac{\pi K}{2}}-\frac{1}{3}\right)+o(1) & \rho=1 \\ \leq \frac{\rho \alpha(1-\rho)}{1-\alpha}+o(1) & \rho<1 .\end{cases}
$$

\section{ConClusion}

In this paper we applied Takacs' generalized ballot theorem to analyze the queue occupancy in a statistical multiplexer in ATM networks for a special type of burst-constrained traffic, periodic interchangeable (PI) traffic. Explicit formulas for the expected queue occupancy are derived for special PI traffic: UPI and UUPI. The result can shed light on the study of the worst case performance of a statistical multiplexer with burst-constrained traffic in ATM networks.

\section{REFERENCES}

[1] P. Humblet, A. Bhargava, and M. G. Hluchyj, "Ballot theorems applied to the transient analysis of $\mathrm{nD} / \mathrm{D} / 1$ queues", IEEE/ACM Trans. on Networking, vol. 1, no. 1, Feb. 1993.

[2] L. Tackas, Combinatorial Methods in the Theory of Stochastic Processes, New York: Wiley, 1967. 